Accepted ver.

European journal of operational research. - ISSN 0377-2217. - Vol. 223, no. 2 (2012): 417-420

doi: 10.1016/j.ejor.2012.06.016

\title{
Acceptable consistency of aggregated comparison matrices in analytic hierarchy process
}

\author{
Petra Grošelj, Lidija Zadnik Stirn \\ University of Ljubljana, Biotechnical Faculty, 1000 Ljubljana, Slovenia
}

\begin{abstract}
The analytic hierarchy process (AHP) is a method for solving multiple criteria decision problems, as well as group decision making. The weighted geometric mean method (WGMM) is appropriate when aggregation of individual judgements (AIJ) is used. This paper presents a new proof which confirms the theorem that if the comparison matrices of all decision makers are of acceptable consistency, then the weighted geometric mean complex judgement matrix (WGMCJM) also is of acceptable consistency. This theorem was presented and first proved by $\mathrm{Xu}$ (2000), but Lin et al. (2008) rejected the proof. We also discuss under what conditions the WGMCJM is of acceptable consistency when not all comparison matrices of decision makers are of acceptable consistency. For this case we determine the upper bound for the consistency ratio of WGMCJM and provide numerical examples.
\end{abstract}

Key words: Multiple criteria analysis; Group decision making; Analytic hierarchy process (AHP); Consistency;

\section{Introduction}

The Analytic Hierarchy Process (AHP), developed by Saaty (1980), is a method for solving a multiple criteria decision problem in which the criteria are compared pair-wise with respect to their importance. It can also be used for group decision making. Forman and Peniwati (1998) described two basic aggregation methods: aggregating individual priorities (AIP) and

Email addresses: petra.groselj@bf.uni-lj.si (Petra Grošelj), lidija.zadnik@bf.uni-lj.si (Lidija Zadnik Stirn) 
aggregating individual judgements (AIJ). The aggregation of individual priorities (AIP) is suitable when the group acts as separate individuals. In this case, a weighted arithmetic mean method (WAMM) is usually used. The aggregation of individual judgements (AIJ) is suitable when the group acts as one individual. Aczel and Alsina (1986) demonstrated that when the importance of the individuals' opinions is not equal, the weighted geometric mean method (WGMM) is the only appropriate method for AIJ. This paper considers the aggregation of individual judgements (AIJ). Xu (2000) proved that if the comparison matrices of all decision makers are of acceptable consistency, then the weighted geometric mean complex judgement matrix (WGMCJM) also is of acceptable consistency. Lin et al. (2008), however, rejected his proof.

It is unfortunate that no further proof of this important theorem is published to the benefit of Xu's or Lin's et al. proof. Thus, in the first part of this paper, we provide such a proof which verifies the Xu's theorem. We believe that this theorem is attractive for group AHP applications. The theorem has already been directly or indirectly used in many applications (Cortés-Aldana et al. (2009), Lee et al. (2009), Rabelo et al. (2007), Sun and Li (2009), Talei and Ali (2008)). In the cases when the researchers have a group of decision makers with individual comparison matrices, they want to know whether the WGMCJM is of acceptable consistency. If all individual comparison matrices are of acceptable consistency, the Xu's theorem and our new proof generated in its support, provide a positive answer.

If not all the individual comparison matrices are of acceptable consistency the WGMCJM should be of acceptable consistency. In the second part of this paper we calculate the upper bound of WGMCJM consistency ratio and discuss the question under what conditions the WGMCJM could be of acceptable consistency if not all individual comparison matrices are of acceptable consistency. Equation (10) presents the sufficient condition for the WGMCJM to be of acceptable consistency. The necessary condition is of future research.

Section 2 of the paper offers the basic definitions of AHP. The proof of the Xu's theorem is given in Section 3. In Section 4 we discuss the conditions under which the WGMCJM is of acceptable consistency when not all comparison matrices of decision makers are of acceptable consistency. For such cases we determine the upper bound for the consistency ratio of WGMCJM and offer some numerical examples. Section 5 presents some conclusions. 
Table 1: The random index (RI) by Saaty (2001)

\begin{tabular}{ccccccccccc}
\hline $\mathrm{n}$ & 1 & 2 & 3 & 4 & 5 & 6 & 7 & 8 & 9 & 10 \\
\hline $\mathrm{RI}$ & 0 & 0 & 0.52 & 0.89 & 1.11 & 1.25 & 1.35 & 1.40 & 1.45 & 1.49 \\
\hline
\end{tabular}

\section{Basic definitions}

Let $A=\left(a_{i j}\right)$ and $B=\left(b_{i j}\right)$ be $n \times n$ comparison matrices of AHP type, where reciprocity is required. Then $A \circ B$ denotes the Hadamard product of $A$ and $B$, where $(A \circ B)_{i j}=a_{i j} b_{i j}$. Let $A^{\alpha}=\left(a_{i j}^{\alpha}\right)$ for $\alpha \in \mathbb{R} . A=\left(a_{i j}\right)$ is consistent if $a_{i j} a_{j k}=a_{i k}$ for $i, j, k=1, \ldots, n$.

We assume that there are $s$ decision makers, $s \geq 2$, with $n \times n$ comparison matrices $A_{k}$, for $k=1, \ldots, s$. The importance of $k$-th decision maker's opinion is denoted by $\alpha_{k}, \alpha_{k} \in(0,1)$ for $k=1, \ldots, s$ and $\sum_{k=1}^{s} \alpha_{k}=1$. Then their group opinion is presented by the WGMCJM $\bar{A}$, where

$$
\bar{A}=A_{1}^{\alpha_{1}} \circ A_{2}^{\alpha_{2}} \circ \cdots \circ A_{s}^{\alpha_{s}} .
$$

Since the judgements of decision makers are usually not consistent, Saaty (1980) defined the consistency index $C I_{A}$ of $n \times n$ comparison matrix $A$ as

$$
C I_{A}=\frac{\lambda_{A, \max }-n}{n-1}
$$

where $\lambda_{A, \max }$ is the largest eigenvalue of $A$. Saaty (1980) presented the consistency ratio

$$
C R_{A}=\frac{C I_{A}}{R I_{n}}
$$

as the measure of the inconsistency of the comparison matrix $A$, where $R I_{n}$ is the random index of $n \times n$ comparison matrices by Saaty (2001) (Table 1). In general, the comparison matrix $A$ is of acceptable consistency if $C R_{A}<0.1$. In some cases certain authors require that the consistency ratio be less than $5 \%$ for $n=3$ and less than $8 \%$ for $n=4$ (Saaty, 2001).

\section{Aggregation of the comparison matrices of acceptable consis- tency}

Theorem 1. Let $n \times n$ comparison matrices $A_{1}, A_{2}, \ldots, A_{s}$ be of acceptable consistency, $\alpha_{k} \in(0,1), \sum_{k=1}^{s} \alpha_{k}=1$, then the WGMCJM $\bar{A}=A_{1}^{\alpha_{1}} \circ A_{2}^{\alpha_{2}} \circ$ $\cdots \circ A_{s}^{\alpha_{s}}$ is of acceptable consistency. 
Proof. The proof of the Theorem 1 is based on the Theorem 2 of Elsner et al. (1988):

Theorem 2. Let $A_{1}, \ldots, A_{s}$ be nonnegative $n \times n$ matrices, and $\alpha_{1}, \ldots, \alpha_{s}$ positive numbers such that

$$
\sum_{k=1}^{s} \alpha_{k} \geq 1
$$

Then

$$
\rho\left(A_{1}^{\alpha_{1}} \circ \cdots \circ A_{s}^{\alpha_{s}}\right) \leq \rho\left(A_{1}\right)^{\alpha_{1}} \cdots \rho\left(A_{s}\right)^{\alpha_{s}},
$$

where $\rho(A)$ denotes the spectral radius of $A$.

The Perron - Frobenius theorem states that for each matrix $A$ with strictly positive entries, the spectral radius $\rho(A)$ is equal to maximal eigenvalue (the Perron root) of $A$. So Theorem 2 (equation (4)) assures that:

$$
\lambda_{\bar{A}, \max }=\lambda_{A_{1}^{\alpha_{1} \ldots \ldots \circ A_{s}^{\alpha_{s}}, \max }} \leq \lambda_{A_{1}, \max }^{\alpha_{1}} \cdots \lambda_{A_{s}, \max }^{\alpha_{s}} .
$$

Let $\beta>0$ be the threshold of acceptable consistency. Equations (2) and (3) imply the equivalent relation for comparison matrix $A$

$$
\left(C R_{A}<\beta\right) \Longleftrightarrow\left(\lambda_{A, \max }<\beta \cdot R I_{n} \cdot(n-1)+n\right)
$$

Since $\sum_{k=1}^{s} \alpha_{k}=1$ and comparison matrices $A_{1}, \ldots, A_{s}$ are of acceptable consistency from Theorem 1 applying (6) to (5) implies that $\bar{A}$ is of acceptable consistency, which is the desired outcome.

At this point we need to emphasize that Theorem 1 holds for general matrices, not only for comparison matrices in AHP, where the reciprocity is required. In this case $R I_{n}$ has to be computed for general matrices.

\section{Aggregation of the comparison matrices, where not all the ma- trices are of acceptable consistency}

In this section we discuss the question: if there are $s, s \geq 2$, decison makers with their opinions of different importance, under what conditions is the WGMCJM $\bar{A}$ of acceptable consistency - when some or all of the individual comparison matrices are not of acceptable consistency?

The upper bound for the consistency ratio of WGMCJM $\bar{A}$ follows from (2), (3) and (5): 


$$
C R_{\bar{A}} \leq \frac{\left(\lambda_{A_{1}, \max }\right)^{\alpha_{1}} \cdots\left(\lambda_{A_{s}, \max }\right)^{\alpha_{s}}-n}{(n-1) \cdot R I_{n}}
$$

The lower bound for the consistency ratio of the WGMCJM is in fact 0 .

Here we present three numerical examples. The first example demonstrate the applicability of the upper bound for the consistency ratio of WGMCJM $\bar{A}(7)$. The second example show that it is not necessary that all of the individual comparison matrices are of acceptable consistency for the WGMCJM $\bar{A}$ to be of acceptable consistency. The third example confirms that the upper bound for the consistency ratio of WGMCJM $\bar{A}(7)$ is tight.

\subsection{Example 1}

Let us start with three matrices $(n=3, s=3)$ from Sun and Greenberg (2006):

$$
A_{1}=\left[\begin{array}{ccc}
1 & 3 & 7 \\
\frac{1}{3} & 1 & 6 \\
\frac{1}{7} & \frac{1}{6} & 1
\end{array}\right], A_{2}=\left[\begin{array}{ccc}
1 & 2 & 7 \\
\frac{1}{2} & 1 & 6 \\
\frac{1}{7} & \frac{1}{6} & 1
\end{array}\right], A_{3}=\left[\begin{array}{ccc}
1 & 6 & 9 \\
\frac{1}{6} & 1 & 4 \\
\frac{1}{9} & \frac{1}{4} & 1
\end{array}\right]
$$

The consistency ratios of these three matrices are: $C R_{A_{1}}=0.0961$, $C R_{A_{2}}=0.0311$ and $C R_{A_{3}}=0.1037$. Matrices $A_{1}$ and $A_{2}$ are of acceptable consistency and a matrix $A_{3}$ exceed a little the treshold of acceptable consistency. Let $\alpha_{1}=\alpha_{2}=\alpha_{3}=\frac{1}{3}$. Then $\bar{A}$ is the geometric mean of $A_{1}$, $A_{2}$ and $A_{3}$ :

$$
\bar{A}=\left[\begin{array}{ccc}
1 & \sqrt[3]{36} & \sqrt[3]{441} \\
\sqrt[3]{\frac{1}{36}} & 1 & 2 \sqrt[3]{18} \\
\sqrt[3]{\frac{1}{441}} & \frac{1}{2 \sqrt[3]{18}} & 1
\end{array}\right]
$$

The upper bound for its consistency ratio calculated from (2), (3) and (7) is $C R_{\bar{A}} \leq 0.0768$, which indicates that $\bar{A}$ is of acceptable consistency. This upper bound is close to the real consistency ratio of $\bar{A}$ which is $C R_{\bar{A}}=0.0725$. 


\subsection{Example 2}

This example considers six comparison matrices from Altuzarra et al. (2007):

$$
\begin{aligned}
& B_{1}=\left[\begin{array}{lllll}
1 & 3 & 5 & 4 & 7 \\
\frac{1}{3} & 1 & 3 & 2 & 5 \\
\frac{1}{5} & \frac{1}{3} & 1 & \frac{1}{2} & 3 \\
\frac{1}{4} & \frac{1}{2} & 2 & 1 & 3 \\
\frac{1}{7} & \frac{1}{5} & \frac{1}{3} & \frac{1}{3} & 1
\end{array}\right], B_{2}=\left[\begin{array}{ccccc}
1 & 4 & 3 & 5 & 8 \\
\frac{1}{4} & 1 & 4 & 3 & 6 \\
\frac{1}{3} & \frac{1}{4} & 1 & 1 & 5 \\
\frac{1}{5} & \frac{1}{3} & 1 & 1 & 7 \\
\frac{1}{8} & \frac{1}{6} & \frac{1}{5} & \frac{1}{7} & 1
\end{array}\right], B_{3}=\left[\begin{array}{ccccc}
1 & \frac{1}{2} & 3 & 2 & 5 \\
2 & 1 & 5 & 1 & 2 \\
\frac{1}{3} & \frac{1}{5} & 1 & 2 & \frac{1}{2} \\
\frac{1}{2} & 1 & \frac{1}{2} & 1 & 5 \\
\frac{1}{5} & \frac{1}{2} & 2 & \frac{1}{5} & 1
\end{array}\right], \\
& B_{4}=\left[\begin{array}{ccccc}
1 & 3 & 5 & 2 & 6 \\
\frac{1}{3} & 1 & 1 & 3 & 2 \\
\frac{1}{5} & 1 & 1 & 4 & 5 \\
\frac{1}{2} & \frac{1}{3} & \frac{1}{4} & 1 & \frac{1}{2} \\
\frac{1}{6} & \frac{1}{2} & \frac{1}{5} & 2 & 1
\end{array}\right], B_{5}=\left[\begin{array}{ccccc}
1 & 2 & 6 & 3 & 3 \\
\frac{1}{2} & 1 & 2 & 5 & 4 \\
\frac{1}{6} & \frac{1}{2} & 1 & \frac{1}{2} & 1 \\
\frac{1}{3} & \frac{1}{5} & 2 & 1 & 5 \\
\frac{1}{3} & \frac{1}{4} & 1 & \frac{1}{5} & 1
\end{array}\right], B_{6}=\left[\begin{array}{ccccc}
1 & 2 & 5 & 4 & 9 \\
\frac{1}{2} & 1 & 3 & 2 & 6 \\
\frac{1}{5} & \frac{1}{3} & 1 & 1 & 2 \\
\frac{1}{4} & \frac{1}{2} & 1 & 1 & 3 \\
\frac{1}{9} & \frac{1}{6} & \frac{1}{2} & \frac{1}{3} & 1
\end{array}\right] .
\end{aligned}
$$

The consistency ratios of these six matrices are $C R_{B_{1}}=0.0285, C R_{B_{2}}=$ $0.1012, C R_{B_{3}}=0.0286, C R_{B_{4}}=0.1482, C R_{B_{5}}=0.1245$, and $C R_{B_{6}}=$ 0.0053. Matrices $B_{1}, B_{3}$ and $B_{6}$ have very small consistency ratios and are of acceptable consistency. The consistency ratios of the other three matrices exceed the threshold of acceptable consistency. Let $\alpha_{k}=\frac{1}{6}$ for $k=1, \ldots, 6$. The inequality (7) assures that the consistency ratio of WGMCJM $\bar{B}$ is less or equal to 0.0715 , which means that $\bar{B}$ is of acceptable consistency. This statement could be easily confirmed by calculating the geometric mean of $B_{1}, \ldots, B_{6}$, i.e., $\bar{B}$ and its consistency ratio.

\subsection{Example 3}

With this example we show that the upper bound of $C R_{\bar{A}}$, assessed by (7), in general, cannot be lower. The following example presents three matrices, for which the equality in (7) holds:

$$
C_{1}=\left[\begin{array}{cccc}
1 & 2 & 1 & \frac{1}{3} \\
\frac{1}{2} & 1 & 1 & 1 \\
1 & 1 & 1 & 2 \\
3 & 1 & \frac{1}{2} & 1
\end{array}\right], C_{2}=\left[\begin{array}{cccc}
1 & 6 & 3 & 1 \\
\frac{1}{6} & 1 & 1 & 1 \\
\frac{1}{3} & 1 & 1 & 2 \\
1 & 1 & \frac{1}{2} & 1
\end{array}\right] \text { and } C_{3}=\left[\begin{array}{cccc}
1 & 3 & 3 & 1 \\
\frac{1}{3} & 1 & 2 & 2 \\
\frac{1}{3} & \frac{1}{2} & 1 & 2 \\
1 & \frac{1}{2} & \frac{1}{2} & 1
\end{array}\right]
$$

The consistency ratios of these three matrices are: $C R_{C_{1}}=C R_{C_{2}}=$ $C R_{C_{3}}=0.1823$. Let $\alpha_{1}=\alpha_{2}=\alpha_{3}=\frac{1}{3}$. The geometric mean of $C_{1}, C_{2}$ and 
$C_{3}$ is

$$
\bar{C}=\left[\begin{array}{cccc}
1 & \sqrt[3]{36} & \sqrt[3]{9} & \sqrt[3]{\frac{1}{3}} \\
\sqrt[3]{\frac{1}{36}} & 1 & \sqrt[3]{2} & \sqrt[3]{2} \\
\sqrt[3]{\frac{1}{9}} & \sqrt[3]{\frac{1}{2}} & 1 & 2 \\
\sqrt[3]{3} & \sqrt[3]{\frac{1}{2}} & \frac{1}{2} & 1
\end{array}\right]
$$

The consistency ratio of $\bar{C}$ is $C R_{\bar{C}}=0.1823$ which is equal to its upper bound from (7).

The condition

$$
\frac{\left(\lambda_{A_{1}, \max }\right)^{\alpha_{1}} \cdots\left(\lambda_{A_{s}, \max }\right)^{\alpha_{s}}-n}{(n-1) \cdot R I_{n}}<\beta,
$$

where $\beta$ is the threshold of acceptable consistency, is a sufficient condition for the WGMCJM to be of acceptable consistency, but it is not a necessary condition.

\section{Conclusions}

This paper contains the new proof that if all individual comparison matrices are of acceptable consistency then the WGMCJM is of acceptable consistency. In addition, we showed that it is not neccessary for all individual comparison matrices to be of acceptable consistency for the WGMCJM to be of acceptable consistency. The upper bound for the consistency ratio of WGMCJM, which is based on the largest eigenvalues (or equivalently on consistency ratios) of given comparison matrices and the importance of the decision makers' opinions, is given with equation (7).

With Examples 1, 2, and 3 we demonstrated that the WGMCJM still may be of acceptable consistency even if not all decision makers' comparison matrices are of acceptable consistency.

Since the consistency ratio of the WGMCJM can be lower than the given upper bound it doesn't depend only on the consistency ratios and the importance of the opinions of all decision makers, but also on the individual's judgements in their comparison matrices. Further research could investigate these relations. 


\section{References}

J. Aczel, C. Alsina. On synthesis of judgements. Socio-Economic Planning Sciences 1986;20; 333-339.

A. Altuzarra, J. M. Moreno-Jiménez, M. Salvador. A Bayesian priorization procedure for AHP-group decision making. European Journal of Operational Research 2007;182;367-382.

F. A. Cortés-Aldana, M. García-Melón, I. Fernández-de-Lucio, P. AragonésBeltrán, R. Poveda-Bautista. University objectives and socioeconomic results: A multicriteria measuring of alignment. European Journal of Operational Research 2009;199; 811-822.

L. Elsner, C. R. Johnson, J. A. Dias Da Silva. The Perron root of a weighted geometric mean of nonnegative matrices. Linear and Multilinear Algebra $1988 ; 24 ; 1-13$.

E. Forman, K. Peniwati. Aggregating individual judgements and priorities with the Analytic Hierarchy Process. European Journal of Operational Research 1998;108; 165-169.

A. H. I. Lee, H.-J. Chang, and C.-Y. Lin. An evaluation model of buyersupplier relationships in high-tech industry - The case of an electronic components manufacturer in Taiwan. Computers \& Industrial Engineering 2009;57; 1417-1430.

R. Lin, J. Shu-Jen Lin, J. Chang, D. Tang, H. Chao, P. C Julian. Note on group consistency in analytic hierarchy process. European Journal of Operational Research 2008;190; 672-678.

L. Rabelo, H. Eskandari, T. Shaalan, M. Helal. Value chain analysis using hybrid simulation and AHP. International Journal of Production Economics 2007;105; 536-547.

T.L. Saaty. Multicriteria Decision Making: The Analytic Hierarchy Process. McGraw-Hill: New York; 1980.

T.L. Saaty. Fundamentals of Decision Making and Priority Theory with the Analytic Hierarchy Process. RWS Publications: Pittsburgh; 2001. 
J. Sun, H. Li. Financial distress early warning based on group decision making. Computers \& Operations Research 2009;36; 885-906.

L. Sun, B. S. Greenberg. Multicriteria Group Decision Making: Optimal Priority Synthesis from Pairwise Comparisons. Journal of Optimization Theory and Applications 2006;130;317-338.

M. Taleai, M. Ali. Using delphi-AHP method to survey major factors causing urban plan implementation failure. Journal of Applied Sciences.2008;8; $2746-2751$.

Z. Xu. On consistency of the weighted geometric mean complex judgement matrix in AHP. European Journal of Operational Research 2000;126; 683687. 